\title{
Professor T.H. Lin Memorial Issue in The International Journal of Damage Mechanics
}

\section{Guest Editors: Lizhi Sun and J. Woody Ju}

The Professor T.H. Lin Memorial Issues, published in the May and July issues of the International Journal of Damage Mechanics (IJDM), are motivated by the special Symposium entitled 'T.H. Lin 95th Birthday Symposium on Computational Mechanics and Materials' as part of the 7th World Congress on Computational Mechanics, held on July 16-22, 2006, in Los Angeles, California. The selected papers in these special IJDM issues encompass a variety of state-of-the-art mechanical and damage formulations and computational implementations for engineering materials, paying tribute to Professor T.H. Lin for his important contributions in the field of mechanics and materials.

We would like to express our gratitude to the authors for contributing their quality work to these memorial journal issues. All papers have been rigorously peer-reviewed following the standard IJDM review criteria. Furthermore, we express our sincere appreciation to IJDM Editor Professor C.L. Chow for his support of the concept of these special issues.

Lizhi Sun, Associate Professor Department of Civil and Environmental Engineering University of California Irvine, CA 92697-2175

Jiann-Wen Woody Ju, Professor Department of Civil and Environmental Engineering University of California Los Angeles, CA 90095-1593 